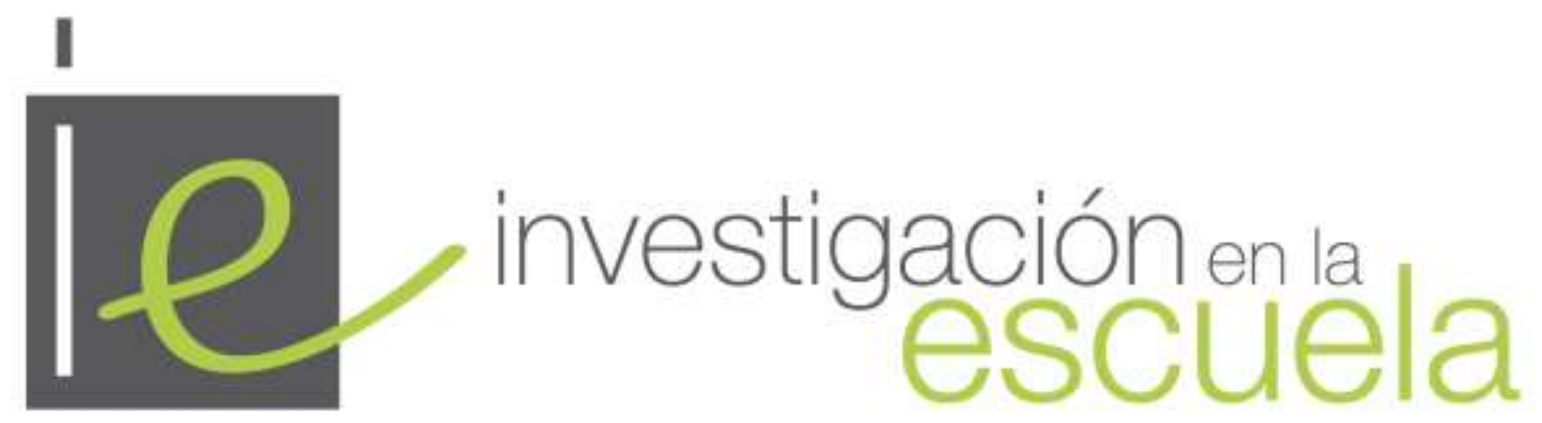

Revista internacional de investigación e innovación educativa

\title{
La innovación en la enseñanza de las ciencias también es una cuestión política e ideológica II: La evolución del Proyecto IRES
}

\author{
Gabriela Cattani Delord", Rafael Porlán ${ }^{1}$ y Gleny Duro Guiarães ${ }^{2}$ \\ Universidad de Sevilla $^{1}$, Pontificia Universidad Católica de Río Grande del Sur ${ }^{2}$ \\ España, Brasil
}

Citación: Delord, G. C, Porlán, R. y Duro Guiarães, G. (2018). La innovación en la enseñanza de las ciencias también es una cuestión política e ideológica. II: La evolución del Proyecto IRES. Investigación en la Escuela, 96, 1-15.

Resumen: Este artículo, el segundo de una serie de dos, completa el estudio del discurso innovador del Proyecto IRES (Investigación y Renovación Escolar). En el anterior se analizó su origen y fundamentos, especialmente en el ámbito de la enseñanza de las ciencias, y en este se investiga su evolución a lo largo de sus 30 años de existencia. Se han tomado como fuentes de información las entrevistas a los fundadores y varios documentos de referencia. El método de análisis se ha basado en el Análisis del Discurso (AD) y los resultados ponen de manifiesto que el proyecto, en este tiempo, no ha conseguido la generalización esperada. Su ideología alternativa, puesta de manifiesto en el primer artículo, choca con el discurso neoliberal, lo que ha llevado a sus miembros a intervenir activamente en el debate social y político sobre el modelo de escuela.

Palabras clave: "Innovación"; "análisis del discurso"; "enseñanza de las ciencias"; "proyecto IRES"; "investigación en la escuela”. 
Innovation in the science education is also a political and ideological issue. II: The evolution of the IRES Project

Abstract: This article, the second of a series of two, completes the study of the innovative discourse of the IRES Project (Research and Renewal of the School). In the previous one, its origin and foundations were analyzed, especially in the field of science education, and in this the evolution is investigated throughout its 30 years of existence. The interviews with the founders and several reference documents have been taken as sources of information. The method of analysis has been based on the Discourse Analysis (AD) and the results show that the project, at this time, has not achieved the expected generalization. Its alternative ideology, highlighted in the first article, clashes with the neoliberal discourse, which has led its members to actively intervene in the social and political debate on the school model.

Key words: "Innovation"; "discourse analysis"; "science education"; "IRES project"; "school research".

\section{L'innovation dans l'enseignement des sciences est aussi une question politique et idéologique. II: L'évolution du projet IRES}

Resumè: Cet article, le deuxième d'une série de deux, complète l'étude du discours novateur du projet IRES (Recherche et Renouvellement de l'École). Dans le précédent, son origine et ses fondements ont été analysés, en particulier dans le domaine de l'éducation scientifique, et ici l'évolution est étudiée tout au long de ses 30 années d'existence. Les entretiens avec les fondateurs et plusieurs documents de référence ont été pris comme sources d'information. La méthode d'analyse a été basée sur l'analyse du discours (AD) et les résultats montrent que le projet, à ce jour, n'a pas atteint la généralisation attendue. Son idéologie alternative, mise en évidence dans le premier article, se heurte au discours néolibéral, qui a conduit ses membres à intervenir activement dans le débat social et politique sur le modèle scolaire.

Mots-clés: "Innovation"; "analyse du discours"; "éducation scientifique"; "projet IRES"; "recherche scolaire".

\section{Introducción}

Este artículo es la continuación de otro publicado en esta misma revista (Delord, Porlán y Guimaraes, en prensa). En aquel argumentábamos que todo discurso, también el pedagógico, tiene un trasfondo ideológico que recibe influencias de la historia, del contexto político y social, de otros discursos y de la experiencia personal. Como ejemplo de esto centramos nuestro análisis en el caso del Proyecto IRES -en adelante P. IRES- (Grupo Investigación en la escuela, 1991) y del Modelo de Investigación en la Escuela (MIE) que lo sustenta, y utilizamos como referente teórico y metodológico la teoría del Análisis del Discurso (AD) de Pêcheux (1995).

También analizamos que los discursos innovadores en la enseñanza de las ciencias (EC) (y por extensión, en la escuela en general) pueden ser clasificados en dos corrientes distintas:

a) Los que tienen fines vinculados al cambio social y que confrontan explícitamente con la ideología pedagógica dominante, que hemos denominado Innovadores/Alternativos, como es el caso del P. IRES.

b) Los que reducen el problema del cambio a un asunto metodológico, técnico o/y profesional sin cuestionar los fines ocultos de la cultura escolar dominante, que hemos denominado Innovadores/Neoliberales. 
IRES

En este segundo artículo pretendemos analizar cómo el P. IRES se ha desarrollado y posicionado a lo largo del tiempo en el contexto de la escuela tradicional conservadora, y de su variante neoliberal de tiempos más recientes.

\section{Marco teórico}

No vamos a repetir el marco de referencia general de este trabajo que ya desarrollamos en el artículo anterior, pero sí nos detendremos, siguiendo a Saviani (1994), Illich (1971), Laclau y Mouffe (1985) y Merchán (2012), en analizar las contradicciones existentes entre el discurso idealizado de la EC de la escuela tradicional y el discurso oculto y real de la misma (Torres, 1991). Hemos insistido en que la escuela mayoritaria es caracterizada por Althusser (1989) como un aparato ideológico que promueve objetivos tácitos en relación con la domesticación de la ciudadanía. Desde nuestro punto de vista, esta función de control y adiestramiento social se realiza, entre otras, a través de las estrategias siguientes (Delors, 2003):

a) Educando para atender las necesidades del sistema político y económico dominante y no para promover el desarrollo de una ciudadanía alfabetizada científicamente, consciente y comprometida.

b) Enseñando contenidos científicos fragmentados y desvinculados de los problemas relevantes de nuestro mundo, no promoviendo, así, el enriquecimiento del saber ciudadano común.

c) Pretendiendo enseñar por transmisión directa de información, provocando la sumisión a un saber externo y especializado.

d) Tratando de calificar el aprendizaje mecánico del estudiante, lo que provoca su condicionamiento conductual y una selección social muy vinculada al origen de clase.

Estas estrategias han favorecido históricamente la formación de ciudadanos competitivos y oprimidos, según Campbell y Neill (1994) y Freire (1981), así como el mantenimiento de una sociedad poco libre, justa, igualitaria y sostenible, como nos indican Bacharach y Mundell (1993) y Ball (1987).

Además, Adorno (1973), Anderson y Grinberg (1998) y Argyris y Schön (1974) describen que la escuela tradicional, su currículo, su modelo de enseñanza/aprendizaje y su forma de evaluación enjuiciadora y sancionadora se posiciona como si todos los alumnos fueran iguales, cuando son diferentes, sancionando y excluyendo a los que no se adaptan a los patrones establecidos. Además, los espacios, los tiempos y los comportamientos considerados deseables están pensados para mantener al alumno controlado dentro de la escuela y para que en el futuro sea, en gran parte, un asalariado adaptado a la producción de capital (Dewey, 1995; Foucault, 2004; Habermas, 1975; Hargreaves, 1994).

Saviani (1994), en esta misma línea, afirma que la escuela desarrolla valores que atienden muy bien a los objetivos del capital, pero que su discurso está disfrazado de una visión idealizada y engañosa (Figura 1). De acuerdo con este autor y con Cuesta (2004), la sociedad contemporánea crea un falso espejismo de lo que la escuela representa. Se entiende que a través de la educación todos tendrán acceso a buenos trabajos, a la realización personal y a la libertad de consumo. Sin embargo, la escuela tradicional realmente trata de asegurar un gran número de alumnos (futuros trabajadores) con un mínimo de conocimientos y un máximo de lagunas epistemológicas para, así, atender sin problemas a la producción de capital. En definitiva, la escuela sigue siendo una herramienta potente para formar trabajadores útiles al sistema, reproduciendo las pautas de pensamiento y de acción convencionales que mantienen la cultura social dominante (Apple, 2006). 


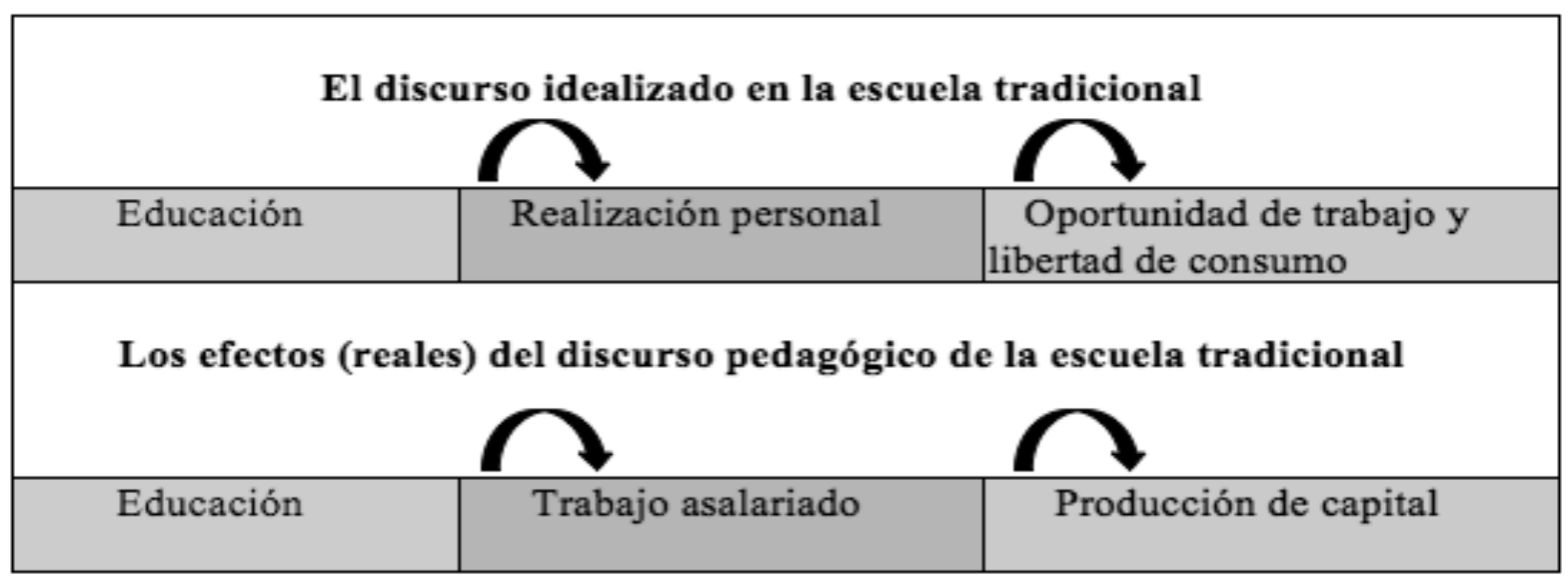

Figura 1. El discurso idealizado de la escuela tradicional versus su discurso real.

\section{Metodología}

Si en el artículo primero analizamos el origen, las influencias y los fundamentos del MIE y del P. IRES el reto de este es analizar cómo los fundadores y el colectivo Foro por Otra Escuela de Sevilla al que pertenecen, como parte de la red que ha desarrollado el proyecto (Red IRES), se ha ido posicionado a lo largo del tiempo en el contexto pedagógico dominante. Las cuestiones centrales, por tanto, son:

¿Cómo desde el P. IRES se ha intentado favorecer e impulsar el cambio en la enseñanza de las ciencias y en la escuela?

¿Cuáles han sido los obstáculos que ha tenido el proyecto para extenderse y generalizarse?

¿Cuáles han sido las estrategias utilizadas para sortear dichos obstáculos y hacer avanzar el P. IRES a lo largo de los años?

Esta segunda parte del análisis se ha basado en las entrevistas semiestructuradas a los fundadores, pero utilizando las unidades de información relacionadas con las cuestiones mencionadas. Sin embargo, los tres artículos que se utilizaron como fuentes de información en el artículo primero han sido sustituidos por diversos documentos que ilustran la evolución del proyecto: El Manifiesto Pedagógico No es Verdad (Doc.1, www.redires.net), el manifiesto La Escuela que Proponemos (Doc.2, Porlán et al., 2014) y el proyecto municipal Sevilla: Ciudad Educadora (Doc.3, García Pérez, Porlán, Chica y Gordillo, 2017).

El tratamiento de estas fuentes está descrito en este mismo apartado del artículo anterior (Delord, Porlán y Guimaraes, en prensa). Los recortes discursivos o unidades de información fueron codificados con la letra A cuando se obtuvieron de las transcripciones de las entrevistas, seguida del número correspondiente a cada autor (1, 2, 3 y 4) o con las letras Doc cuando se obtuvieron de los documentos, seguidas también del número de orden de cada uno (1, 2 y 3). Por razones de espacio se muestran a continuación solo una parte de los recortes discursivos seleccionados para cada Posición Sujeto identificada. 
IRES

\section{Análisis discursivo}

\section{Posición Sujeto 1: La generalización del cambio de la escuela contando con apoyo institucional}

El Modelo de Investigación en la Escuela, cuyo origen es anterior a la aparición de los documentos programáticos del P. IRES, tuvo mucho éxito en la década de los 80 , cuando los profesores y la sociedad estaban motivados con el fin del franquismo y con los cambios que se estaban produciendo. En este clima de entusiasmo, los autores estaban convencidos de la potencialidad del modelo y de la posibilidad de ir cambiando el discurso escolar conservador por otro alternativo y democrático siguiendo una estrategia a modo de mancha de aceite.

El M.I.E tiene mucha potencialidad y versatilidad (...) Se puede comprobar cuando con los mismos principios podemos trabajar en programas de Educación Ambiental, de Educación para la Ciudadania, en propuestas en Infantil, Primaria y Secundaria, en la formación inicial y permanente del profesorado, en la universidad... (A.4, PS1).

Tuvimos en su momento mucho éxito, los profesores de infantil, primaria y secundaria nos buscaban. En nuestras Jornadas de Investigación en la Escuela no teníamos sillas para todos (A.2, PS1).

A mi no me preocupaba el número de profesores del proyecto, pues creía que si aplicábamos bien el MIE ibamos a tener resultados fantásticos con los estudiantes y contaminaríamos a otros colegas y, con el tiempo, la escuela estaría curada del cáncer de la enseñanza tradicional (A.4, PS1).

Lo que llamamos la estrategia de la mancha de aceite; creíamos que este modelo se desarrollaría poco a poco y que se extendería en las escuelas sobrepasando el poder de la escuela tradicional (A.1, PS1).

El intento más consistente de generalizar las propuestas del MIE fue en el marco del movimiento de experimentación curricular que se puso en marcha institucionalmente en España a raíz del primer gobierno socialista, como preludio a la elaboración de la LOGSE (Ley Orgánica del Sistema Educativo) en 1990. En Andalucía, los fundadores del proyecto tuvieron un papel esencial en este movimiento. Se trataba de que una serie de centros pusieran a prueba un currículo experimental, con la participación de la comunidad educativa. El proceso no solo abarcó las experiencias en EC, sino que transformó la estructura curricular de todas las áreas y la distribución de espacios y tiempos. Duró 4 años, y el último, en 1987, cuando ya participaban 100 centros, culminó en un congreso donde se aprobó la Propuesta Curricular para Andalucía, elaborada y experimentada desde los centros, y un manifiesto con medidas complementarias relativas a la autonomía de los centros, a las dotaciones presupuestarias y a los salarios de los docentes. Este manifiesto provocó el rechazo de los responsables gubernamentales que consideraron excesivamente radical y ambicioso el movimiento que ellos mismos habían impulsado. Procesos similares se dieron en el conjunto del país, de tal manera que finalmente la nueva ley (LOGSE) se cerró con la ayuda de expertos universitarios sin tener en cuenta los resultados de la experimentación, provocando enormes frustraciones en sectores importantes de la comunidad educativa.

Todos nosotros estuvimos implicados en el proceso de experimentación de Andalucía, muy abierto pero a la hora de ponerlo en práctica no funcionó como esperábamos. Para su generalización queríamos que en cada escuela bubiera un profesor asesor, pero no se dio dinero para eso. Se colocó uno para caza zona abarcando bastantes colegios. Con el tiempo, el proceso se fue olvidando y acabando. (A.2, PS1).

En el 86, comienza a organizarse una reforma educativa fuerte, impulsada por el partido socialista (...), eso nos dio la oportunidad de influir en todo ese proceso, yo mismo fui Director del Centro de Profesores de Sevilla. También estuvimos en el equipo central de la reforma en Andalucía. ¿Cuál fue el problema? La tentativa fracasó (...). La idea inicial del Partido Socialista era hacer una reforma contando con los profesores, pero después se valoró que el proceso era muy lento y decidieron juntar a un 
grupo de catedráticos en Madrid (...) e hicieron el Libro Blanco de la reforma de arriba a abajo, y nos desvinculamos. La idea del grupo de catedráticos era promover el constructivismo de arriba a abajo y la nuestra era que tenía que ser construido a partir de la base (...). Fue nuestro primer intento de extender las ideas del IRES, pero fue un fracaso porque las visiones políticas no lo permitieron (A.3, PS1).

Todo empieza cuando entra el partido socialista en el poder, después de la dictadura. Proponen una estrategia de abajo a arriba. El Ministro de Educación invitó a los colectivos pedagógicos a participar en un proceso de experimentación durante cuatro años. Propusieron que antes de cambiar la ley se experimentara el curriculo en las escuelas. En Andalucía se aplicó con la implicación de nuestro grupo, (...) Primero se empezó con la Primaria y luego con la Secundaria. Yo estaba en la coordinación y cada año más escuelas se implicaban. Se trabajaba a través de la investigación de problemas (lo que boy parece una novedad anglosajona). Las escuelas tenian tres horarios: uno dedicado a los proyectos de investigación, otro a talleres para elaborar recursos para la investigación y un tercero para trabajar la Lengua y las Matemáticas, especialmente con los estudiantes más retrasados. ¿Qué ocurrió? Pasados los cuatro años hubo un congreso en el que participaron todas las escuelas del movimiento y se elaboró La Propuesta Curricular para Andalucía y se entregó al gobierno. Unos meses después la administración socialista se asustó porque era una propuesta muy ambiciosa, que incluía también la mejora de salario de los profesores, de cambios arquitectónicos para las escuelas, autonomía para los centros... y rompió el acuerdo (A.4, PS1).

Cuando los políticos se enteraron de lo lento y difícil que es cambiar la escuela, que no es solo una cuestión curricular, sino también de formación docente, de mejora del salario, de cambios en las infraestructuras, pues rompen lo que tenían prometido y llaman a un par de catedráticos y desarrollan a través de algunas reuniones el curriculum escolar de arriba abajo, ignorando todas las propuestas colectivamente desarrolladas (A.4, PS1).

Como podemos ver, por lo que dicen los fundadores, la visión cortoplacista y limitada de los políticos socialistas y las limitaciones económicas con las que querían realizar la reforma educativa, fueron los obstáculos que impidieron continuar con un proceso que despertó el entusiasmo y la participación de muchas escuelas.

\section{Posición Sujeto 2: La estrategia de la mancha de aceite de forma autónoma y la evidencia de su fracaso}

A principios de los años noventa se formaliza el P. IRES y empieza a desarrollar sus principios a través de sus propios colectivos de forma autónoma y sin compromisos institucionales. Debido a la actuación de los responsables políticos en el proceso de reforma institucional, los fundadores creyeron que el IRES, con sus medios, haría posible la estrategia de mancha de aceite en la que aún creían, y que más profesores se unirían a los colectivos y que este cambio sería posible sin ayuda del gobierno. Sin embargo, los años van pasando y el número de profesores implicados en el proyecto va disminuyendo. Al mismo tiempo, cada vez más ellos se implican en sus trabajos académicos en la universidad. Finalmente, acaban reconociendo el fracaso de la estrategia soñada y analizan las causas del mismo.

Creo que por muchas razones la mancha de aceite en la que creíamos no ocurrió. Cada año el número de personas implicadas fue disminuyendo... (A.1, PS2).

Yo pensaba que sería como una mancha de aceite que se iría extendiendo, y eso no sucedió (A.3, PS2).

... pasamos por momentos de mucho entusiasmo, de mucha motivación, pero la situación ha ido cambiando, creo que es evidente: ahora hacemos reuniones con 50, 60 personas y entonces teníamos más de mil personas en los encuentros de Sevilla (A.3, PS2).

El IRES es una buena propuesta, pero en un contexto con muchos condicionantes tiene dificultades para desarrollarse. Porque nosotros solo controlamos algunos. No controlamos los espacios y los tiempos de las escuelas, las cuestiones administrativas, el contexto familiar y social... (A.1, PS2). 
IRES

Con la experiencia del gobierno de Andalucía ya vi que la cuestión educativa es difícil de generalizar, la innovación puede cambiar a un profesor, pero no puede extenderse la fábula de la mancha de aceite sin contar con que existen muchos obstáculos (A.1, PS2).

¿Cómo es posible que el P. IRES que es tan potente se haya reducido a solo 50 personas? Lo digo como una autocrítica, pues creo que muchos de nosotros nos hemos dedicado en exceso a los problemas académicos (A.1, PS2).

Creo que por culpa de todos dejamos la práctica en la escuela y nos centramos más en la universidad. No digo culpa, más bien fue el contexto. Eso ba sido un fallo nuestro (A.1, PS2).

También aparecen otras causas más relacionadas con las presiones del contexto escolar.

En la universidad estábamos en minoría, como en las escuelas y pasamos a ser controlados. Muchos alumnos se ponían a favor nuestra y nos enseñaban grabaciones de profesores dictadores. Lo mismo ocurre en los coles, hasta hoy, porque la innovación produce rechazo. Es complicado ser innovador (A.2, PS2).

Las escuelas tienen una cultura contraria a la innovación: la estructura, los horarios, los demás docentes.

Hay que estar junto a los profesores que quieren hacer innovación y no hemos podido atender a todos (A.3, PS2).

$\mathrm{Y}$ aparecen también reflexiones sobre el contexto social neoliberal y su influencia en el mantenimiento de la escuela tradicional.

El problema es que el cambio social es lento (...). Yo creo que el modelo pedagógico dominante sigue siendo dominante por una cuestión social, y los profesores son parte de la sociedad (A.2, PS2).

Hay que tener en cuenta que muchos de los autores del P. IRES son hijos de la dictadura, muchos profesores de boy no saben lo que es eso. Son de la cultura del neoliberalismo y están conformes con eso, así que cambiar a esta nueva generación es complicado. Las personas de esta generación no son críticas, viven la crisis económica como si fuera algo personal y no social, es complicado hacer el cambio (A.2, PS2).

... el problema es que nosotros hablamos de constructivismo, de lo colectivo, de lo social, y hoy tenemos una sociedad competitiva e individualista, cambiar esta ideología es complicado, lo que se dice en nuestra teoría está lejos de la práctica social habitual (A.2, PS2).

El problema es que surge el neoliberalismo y desde mi punto de vista ha babido una regresión en la sociedady las ideas del IRES chocan con el neoliberalismo (A.2, PS2).

Un modelo pedagógico innovador necesita ir en paralelo con el cambio social. No esperábamos que el modelo neoliberal fuera el modelo dominante (A.2, PS2).

Las familias tienen una representación social sobre la enseñanza y el aprendizaje $y$ quieren que sus hijos hagan las cosas rápido, aprendan a leer muy rápido, no les importa cómo, no están de acuerdo con que cada alumno tiene su tiempo (A.4, PS2).

La sociedad tarda mucho para cambiar una idea. Nosotros siempre hemos estado luchando solos y lo mismo pasa con los profesores innovadores que están solos, algunos se sienten tan solos que vuelven al modelo tradicional, por eso es necesario también cambiar el pensamiento social (A.2, PS2).

La idea de la enseñanza tradicional es tan potente y está tan enraizada en la sociedad que las personas creen que aprender es memorizar y enseñar es dictar conocimientos. Los profesores son parte de la sociedad y la sociedad solo conoce una forma de enseñar, por lo tanto, el problema no es solo aplicar un modelo alternativo, sino cambiar la mentalidad social (A.4, PS2).

Como hemos visto, los fundadores toman conciencia de que hay diferentes tipos de obstáculos para desarrollar el P. IRES de forma autónoma: la presión del contexto escolar, la coherencia entre la cultura neoliberal dominante y la escuela tradicional, sus propias tareas académicas, etc., y también de que finalmente la estrategia de la mancha de aceite no funciona. Lo cierto es que cuando asumen las enormes dificultades para cambiar la escuela ya están en el año 
2000. Así que adoptan una nueva posición, la de intervenir en el debate social sobre la escuela para favorecer la toma de conciencia sobre las repercusiones del modelo tradicional.

Posición Sujeto 3: La intervención activa en el debate social sobre la escuela

Una de las acciones más importantes en este sentido es la elaboración, difusión y publicación del Manifiesto Pedagógico No es Verdad en 2009. Los miembros de la red, por iniciativa del Foro por Otra Escuela de Sevilla, lo difunden con gran éxito de apoyos individuales y de colectivos pedagógicos, sociales, sindicales y políticos, publicándose en periódicos relevantes y presentándose en varias ciudades españolas.

Como la enseñanza siempre ha seguido un único modelo, todas las personas piensan que ese es el modelo de enseñar y aprender, por eso los proyectos innovadores fracasan porque la escuela tiene una concepción epistemológica, filosófica e ideológica con la que la mayoría social está de acuerdo, así que el Manifiesto No es verdad ha pretendido poner en evidencia exactamente eso (A.4, PS).

Por lo tanto, los miembros del P. IRES se esfuerzan por cuestionar y difundir los errores de la enseñanza tradicional a través del manifiesto.

No es verdad que en la escuela española predomine un modelo de enseñanza diferente al tradicional.

A pesar de que hay importantes argumentos en contra de la forma tradicional de enseñar, la cultura escolar

dominante sigue basándose en la transmisión directa de contenidos inconexos y, no pocas veces, desfasados e irrelevantes, en el aprendizaje mecánico y repetitivo, en la evaluación selectiva y sancionadora y en la prolongación de la jornada escolar de los menores con abundantes deberes (Doc.1, PS3).

La mejora de la escuela no es básicamente una cuestión de leyes sino de cambio cultural, social y comunitario (Doc.1, PS3).

No es verdad que en la escuela española hayan bajado los niveles de exigencia Cada vez se pretende enseñar más contenidos, con formulaciones más abstractas y en edades más tempranas. Muchos padres y madres no entienden los libros de texto que con frecuencia protagonizan las tardes familiares. Cada vez es más difícil para los docentes acabar el programa del curso. Cada vez. es más pesada la carga académica de los estudiantes (Doc.1, PS3).

Los estudiantes fracasan, precisamente, porque el modelo de enseñanza tradicional, y no otro, no provoca en ellos un aprendizaje de calidad. Esto siempre ha sido así. No entender las explicaciones, no encontrarle sentido a muchos contenidos, estudiar mecánicamente sólo para los exámenes, olvidar rápidamente lo aprendido y tener que empezar desde cero en cada curso, son experiencias compartidas por muchas personas (Doc.1, PS3).

En un mundo globalizado, donde la información circula por internet, donde la comunicación se ha hecho virtual, donde los graves problemas de la bumanidad tienen carácter interdisciplinar, donde las certezas absolutas han desaparecido y nos enfrentamos a un futuro crítico, incierto y complejo, la escuela sigue anclada en contenidos $y$ métodos del pasado (Doc.1, PS3).

No es verdad que los alumnos y alumnas de ahora sean peores que los de antes. Son diferentes, pero no peores. Los niños, niñas y jóvenes de hoy, y los de antes, son el producto de la sociedad en la que viven. Juzgarlos negativamente como colectivo es una forma de ocultar la responsabilidad de la sociedad adulta (Doc.1, PS3).

El desapego de muchos estudiantes hacia la cultura tradicional de la escuela, oculto en otros tiempos debido al carácter autoritario y represivo de la época franquista, se pone de manifiesto hoy de forma más radical. Este desapego, más que confirmar que el alumnado de hoy es peor que el de antes, como muchos creen, es la evidencia más clara del abismo que los separa de la escuela (Doc.1, PS3). 
IRES

\section{La escuela y la universidad necesitan un cambio}

El fracaso escolar no sólo se manifiesta por los que abandonan o suspenden, también por los que aprueban sin haber conseguido un aprendizaje de calidad. El cambio que proponemos no puede venir de la mano del modelo tradicional, como reclaman algunos, ignorando que dicho modelo es el responsable del fracaso actual. Tampoco aplicando políticas neoliberales de mercantilización de lo educativo (...) ni trasladando a la escuela modelos empresariales de planificación y control de calidad (...). Las personas y su educación no son mercancias (Doc.1, PS3).

El cambio ha de venir de la recuperación y actualización de aquellas ideas y experiencias que han demostrado su capacidad transformadora. La Institución Libre de Enseñanza, la Escuela Nueva, la Escuela Moderna, las Misiones Pedagógicas, los Movimientos de Renovación Pedagógica, etc. son, entre otros, algunos ejemplos valiosos de nuestro pasado (Doc.1, PS3).

Después del éxito de difusión de este manifiesto y teniendo en cuenta su carácter fundamentalmente crítico, la Red IRES elabora e impulsa en 2013 otro manifiesto más propositivo, también a propuesta del colectivo de Sevilla, en el que, de forma divulgativa, se sintetizan los principios básicos de la escuela que se propone, así como pautas de acción política para impulsarla. El manifiesto se denomina La Escuela que Proponemos y sigue siendo a día de hoy el texto fundamental que resume las propuestas de la Red en el debate social y político sobre el cambio de la escuela. Presentamos en lo que sigue algunas de las propuestas de acción de este manifiesto:

Sustitución del uso de los libros de texto por materiales didácticos diversos en forma de bibliotecas de aula (Doc.2, PS3).

Disponibilidad de espacios para la experimentación, como buertos escolares, laboratorios, salas de teatro y de proyecciones (Doc.2, PS3).

Sustitución de las calificaciones por informes de evaluación (Doc.2, PS3).

Sustitución de los exámenes memorísticos por actividades de evaluación que impliquen posibilidades de mejora (Doc.2, PS3).

Incorporación de docentes con buenas prácticas a la formación inicial (Doc.2, PS3).

Aumento significativo del número de horas de prácticas en la formación inicial (Doc.2, PS3).

Apoyo a la creación de redes para la innovación, investigación y mejora de la práctica (Doc.2, PS3).

Rebaja de la ratio profesor/alumnado. Como referencia general, 15 alumnos/as por aula (Doc.2, PS3).

Nueva estructura laboral docente: $50 \%$ del tiempo para clases y 50\% para actividades de diseño de materiales, evaluación, investigación, formación y atención a las familias (Doc.2, PS3).

Normativa para los centros de nueva creación que garantice una arquitectura pedagógica (Doc.2, PS3).

Autonomía de los centros para elaborar un horario abierto y flexible (Doc.2, PS3).

Creación del Consejo de Delegados de Clase como órgano de participación y representación estudiantil en los centros (Doc.2, PS3).

Elección democrática por todos los miembros de la comunidad educativa del Equipo Directivo y del Consejo Escolar (Doc.2, PS3).

Creación de los Consejos Locales de Educación como órganos de participación y colaboración de los agentes educativos y sociales de la zona (Doc.2, PS3).

En estos dos manifiestos la posición del P. IRES contra la pedagogía dominante es evidente. Estos documentos muestran una lucha difícil por cambiar la escuela de hoy en el marco de una sociedad neoliberal, donde los valores dominantes son el individualismo, la competitividad, el consumo y la pasividad. También en ellos empiezan a explicitarse posicionamientos claramente políticos que hasta ahora habían permanecidos implícitos, como es el compromiso por una escuela pública, laica, democrática y de calidad. 
La escuela es un derecho de la ciudadanía que no admite privilegios. El estado debe garantizar ese derecho con una red pública y gratuita de centros educativos (Doc.2, PS3).

La escuela pública ha de ser laica, es decir, ha de respetar todas las creencias, consecuente con la declaración universal de los derechos humanos y de los derechos de los menores. La escuela no es un lugar de adoctrinamiento sino de formación de una ciudadanía libre, autónoma, crítica y comprometida (Doc.2, PS3).

Ha de ser democrática. La participación deseada y promovida, el diálogo basado en argumentos, la construcción colectiva de conocimientos y la lucha contra el poder arbitrario, la injusticia y la exclusión han de ser referentes del sistema educativo (Doc.2, PS3).

Ha de ser una escuela de calidad. Pero la calidad no se basa en la obsesión normativa y en el control tecnocrático. Calidad y profesionalidad son dos caras de la misma moneda. La dignificación profesional y social del profesorado es la que debe garantizar la autonomía y la responsabilidad para el desarrollo de una escuela coherente con los avances de la investigación educativa (Doc.2, PS3).

Por último, y como un paso más en el compromiso por promover un cambio social profundo que acompañe al cambio que la escuela necesita, y ante la aparición en la realidad política española de candidaturas municipales de unidad ciudadana y popular como nuevas formas de hacer política, el Foro por Otra Escuela de Sevilla, como parte de la Red IRES, adopta la posición de participar políticamente en el ámbito municipal.

Posición Sujeto 4: La intervención política en candidaturas ciudadanas municipales

Los tres fundadores del P. IRES que siguen en la red y el colectivo al que pertenecen, se implican en candidaturas ciudadanas para las elecciones municipales en Sevilla y en Dos Hermanas. Su posición es poner en marcha los principios del IRES en el ámbito municipal, el más cercano a las escuelas.

El P. IRES actualmente está intentando validar sus ideas divulgando otra escuela por la vía política, por ahi vamos (A.1, PS4).

Actualmente estoy implicado en un movimiento más político y estoy intentando cambiar la escuela ayudando a desarrollar huertos escolares en el municipio donde vivo (A.3, PS4).

Frente a la situación estatal y local aparece un movimiento ciudadano participativo con el que la Red IRES de Sevilla conecta, y que se plantea la necesidad de una educación ciudadana en los procesos de participación democrática y en la toma de decisiones relativas a los grandes problemas de la sociedad de hoy (Doc.3, PS4).

En este sentido, algunos de los principios educativos presentados por el Foro por Otra Escuela en la candidatura ciudadana municipal de Sevilla Ganemos Sevilla en el año de 2014 fueron:

Sevilla puede ser una ciudad educadora; tiene espacios adecuados, recursos, historia, patrimonio y una trayectoria histórica de actividades educativas innovadoras. Resulta indispensable definir en qué y cómo quiere la ciudad ser educadora (Doc.3, PS4).

En cuanto a los programas educadores, se trataria de promover y ofrecer un elenco de actividades transversales (...) a) Alfabetización (incluyendo la alfabetización científica y tecnológica, como divulgación y familiarización con la ciencia y los medios tecnológicos en vinculación con la vida cotidiana). b) Educación para una ciudadanía participativa y solidaria. c) Educación ambiental. d) Educación para el patrimonio. e) Educación para la igualdad de género.f) Educación para una vida saludable, desde un punto de vista físico, emocionaly social (Doc.3, PS4).

Las dos coaliciones municipales en que el colectivo de Sevilla de la Red IRES se implica no ganan las elecciones y una vez más el discurso del P. IRES no consigue ampliar sus cuotas de influencia social y política en una sociedad que se fía más de los partidos convencionales y neoliberales que de ideas más alternativas.

Finamente, los tres fundadores aún implicados y el Foro por Otra Escuela participan en el año de 2017 en la creación del Movimiento Andaluzpor la Escuela Pública (MAEP), en el que, en 
colaboración con colectivos de padres y madres, estudiantes, sindicatos y partidos políticos alternativos han elaborado el documento Acuerdo Social sobre la Escuela Pública, en el que proponen un modelo de escuela muy influido por los postulados del P. IRES y un incremento presupuestario que la haga posible.

A lo largo de este artículo hemos podido ver que el P. IRES tiene una larga trayectoria de lucha frente al discurso ideológico de la escuela tradicional, una lucha que ya dura más de 30 años, con pocos resultados observables de cambios trascendentes en las escuelas españolas, andaluzas y en la sociedad en general. Los cambios han sido siempre puntuales, parciales y limitados. Actualmente el discurso mayoritario en la sociedad es el discurso neoliberal y los discursos alternativos siguen siendo minoritarios. Cómo apunta Pêcheux (2000), la sociedad está fragmentada en dos pensamientos distintos, el dominante y hegemónico y el contra-hegemónico y alternativo. Los colectivos y personas que contribuyen a este último tendrán que seguir luchando, modificando sus posiciones y estrategias, pues la contradicción central de la época es la de un mundo globalizado, sumido en una crisis general, sin otra salida que el impulso de nuevas formas de relación entre humanos y de estos con el conjunto del planeta.

\section{Conclusiones e implicaciones para el cambio de la enseñanza de las ciencias y de la escuela}

Como conclusión general, hemos visto que el discurso del P. IRES es alternativo al de la EC mayoritaria y al de la escuela tradicional, y que sus propuestas a largo plazo no han tenido el éxito esperado por los fundadores. En el marco de una sociedad conservadora/neoliberal los fundadores han mantenido distintas posiciones conforme han ido comprendiendo los obstáculos que se iban presentando. En primer lugar, confiaron en que una estrategia de mancha de aceite apoyada institucionalmente generalizaría el MIE, sin embargo la mentalidad limitada y cortoplacista de los políticos acabó con dicho proceso. Después creyeron que de forma autónoma lo conseguirían, pero las dificultades debidas al predominio social de una imagen idealizada y mitificada de la escuela tradicional les hicieron comprender que debían intervenir con decisión en el debate social. Para ello, junto a los colegas de la Red, pusieron en marcha sendos manifiestos que trataron de impulsar la toma de conciencia ciudadana. En un paso más se decidieron por la intervención política directa en el ámbito municipal con candidaturas que defendían modelos sociales, participativos y ciudadanos coherentes con los principios del IRES, lo que seguramente dejó menos atendido el compromiso directo con los docentes y con la escuela.

En línea con el proceso seguido por el P. IRES, Duarte (2015) ha investigado la evolución de diferentes proyectos innovadores creados en los años 80 en España en el área de la Didáctica de las Ciencias Sociales. Su trabajo muestra que en los años 90 estos proyectos empiezan a diluirse y que entre 2000 y 2008 la mayoría de ellos deja de existir. La investigación también apunta a la crisis de los proyectos en el contexto neoliberal.

Después de este estudio, creemos que para impulsar el cambio escolar es necesario intervenir en los ámbitos político, económico, social y escolar, poniendo el acento en la reflexión sobre la innovación como una praxis colectiva, simultáneamente pedagógica, ideológica y política. En este sentido, apuntamos algunas propuestas que creemos necesarias para impulsar el cambio de la EC y de la escuela:

a) Divulgar que todo discurso innovador no tiene por qué ser auténticamente transformador, pues algunos siguen fomentando la ideología dominante de la escuela tradicional. 
b) Todo discurso pedagógico debe ser analizado desde lo social, lo económico y lo político. Así, los profesores serán más críticos al poner en marcha sus clases y en el momento de seleccionar sus prácticas innovadoras.

c) El cambio educativo y de la enseñanza de las ciencias no puede ser considerado solo una cuestión de nuevas técnicas metodológicas sino también de coherencia con una nueva ideología transformadora.

d) La cuestión política, económica e ideológica de la educación debe estar más presente en las investigaciones científicas.

e) Los programas de formación docente deben impulsar el cambio de las concepciones epistemológicas, políticas e ideológicas de los futuros docentes.

\section{Referencias}

Adorno, T. (1973). The jargon of authenticity. Evanston: Northwestern University Press.

Althusser, L. (1983). Aparelhos ideológicos de estado: nota sobre os aparelhos ideológicos de estado. Rio de Janeiro: Graal.

Anderson, G.L. \& Grinberg, J. (1998). Educational administration as a disciplinary practice: appropriating Foucault's view of power, discourse, and method. Educational Administration Quarterly, 34(3), 329-353.

Apple, M.W. (2006). Ideología e poder. Porto Alegre: Artmed.

Argyris, C. \& Schön, D. (1974) Theory in practice: Increasing professional effectiveness. San Francisco: JosseyBass.

Ball, S. J. (1987). The Micro-politics of the School: Towards a Theory of School Organization. Londres y Nueva York: Methuen.

Bacharach, S. B. \& Mundell, B. L. (1993). Organizational politics in Schools: Micro, macro and Logic of Action. Educational Administration Quarterly, 29(4), 423-452.

Campbell, J. \& Neill, S. (1994). Curriculum at stage one: Teacher commitment and policy failure. England: Longman.

Cuesta, R. (2004). La escolarización de masas: un sospechoso y "feliz" consenso transcultural. Cuadernos de Pedagogía, 334, 125-131.

Delord, G.C., Porlán, R y Guimaraes, G. (en prensa). La innovación en la enseñanza de las ciencias también es una cuestión política e ideológica. I: La génesis y fundamentos del Proyecto IRES. Investigación en la Escuela.

Delors, J. (2003). Educação: um tesouro a descobrir. Brasilia: Edigfies Asa.

Dewey, J. (1995): Democracia y educación. Madrid: Morata.

Duarte, O. M. (2015). La enseñanza de la historia en la educación secundaria: innovación, cambio y continuidad (Tesis Doctoral). Universidad de Sevilla, Sevilla. Recuperado de https://idus.us.es/xmlui/bitstream/handle/11441/30778/1.\%20TESIS\%20DOCTORAL Du arte $\% 20 \mathrm{Pi} \% \mathrm{C} 3 \% \mathrm{~B} 1 \mathrm{a}$ Olga $\% 20 \mathrm{M} \% \mathrm{C} 2 \% \mathrm{AA}$ 16.07.2015.pdf?sequence=1

Freire, P. (1981). Pedagogía do oprimido. Rio de Janeiro: Paz e Terra.

Foucault, M. (2004). Microfísica do poder. (19 ed.). Rio de Janeiro: Edição Graal.

García Pérez, F.F., Porlán, R., Chica, M.J. y Gordillo, I. (2017). Por una ciudad educadora. Un programa educativo alternativo para la ciudad de Sevilla. En VIII Encuentro iberoamericano de colectivos y redes de maestros y maestras que hacen investigación desde su escuela y comunidad. Michoacán, México.

Grupo Investigación en la Escuela. (1991). Proyecto curricular IRES. Doc. I, II, III, IV. Sevilla: Díada. Habermas, J. (1975). Connaissance et intérêt. Paris: Gallimard. 
IRES

Hargreaves, A. (1994). Changing teachers, changing times: Teachers' work and culture in the postmodern age.

New York: Teachers College Press.

Illich, I. (1971). Deschooling society. New York: Row and Harper.

Laclau, E. \& Mouffe, C. (1985). Hegemony and socialist strategy: Towards a radical democratic politics. London: Verso.

Merchán, J. (2012). La introducción en España de la política educativa basada en la gestión empresarial de la escuela: el caso de Andalucía. Archivos Analíticos de Políticas Educativas, 20(1), 1 28. DOI: http://dx.doi.org/10.14507/epaa.v20n32.2012

Pêcheux, M. (1995). Semântica e discurso: uma crítica à afirmação do óbvio. Campinas: Unicamp.

Porlán, R., Fernández, N., Chica, M., Estepa, J., García Pérez, F.F., Gordillo, I. y Torres, I. (2014). La escuela que queremos. En VII Encuentro iberoamericano de colectivos y redes de maestros y maestras que hacen investigación desde su escuela y comunidad. Cajamarca, Perú.

Saviani, D. (1994). Contribuições da filosofia para a educação. Em aberto, 45(9), 3-9.

Torres, J. (1991). El currículum oculto. Madrid: Morata.

\section{Información sobre los autores}

Autor: Gabriela Cattani Delord

Institución: Universidad de Sevilla

Email: gcattani1@us.es

Autor: Rafael Porlán

Institución: Universidad de Sevilla

Email: rpolan@us.es

Autor: Gleny Duro Guiarães

Institución: Pontifícia Universidade Católica do Rio Grande do Sul

Email: gleny@pucrs.br 


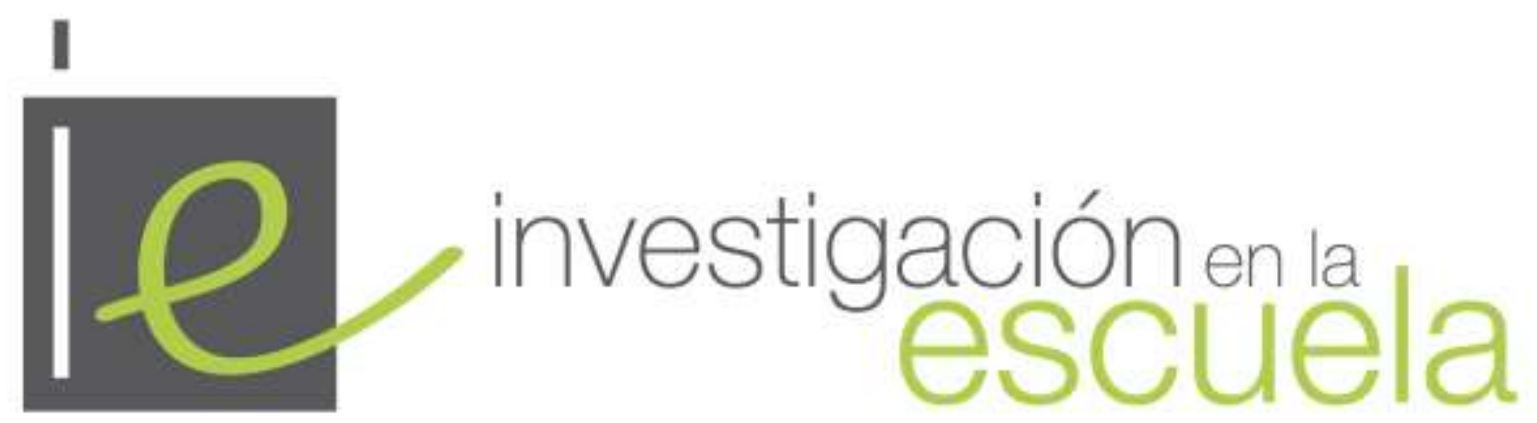

Revista académica evaluada por pares y de acceso abierto

Número 96

31 de diciembre de 2018

ISSN 2443-9991

\section{(C)}

SOMREFIIGHISRESERVEDLOS/as lectores/as pueden copiar, mostrar, y distribuir este artículo, siempre y cuando se de crédito y atribución al autor/es y a Investigación en la Escuela, se distribuya con propósitos no-comerciales, no se altere o transforme el trabajo original. Más detalles de la licencia de CreativeCommons se encuentran en http://creativecommons.org/licenses/by-nc-sa/3.0 Cualquier otro uso debe ser aprobado en conjunto por el autor/es, o Investigación en la Escuela.

ำ investigacion-en-la-escuela

Contribuya con comentarios y sugerencias en la web de la revista. Por errores y sugerencias contacte a secretaria@investigacionenlaescuela.es 


\section{Investigación en la escuela}

Consejo de dirección: Ana Rivero García (Universidad de Sevilla), Nicolás de Alba Fernández (Universidad de Sevilla), Pedro Cañal de León (Universidad de Sevilla), Francisco F. García Pérez (Universidad de Sevilla), Gabriel Travé González (Universidad de Huelva), Francisco F. Pozuelos Estrada (Universidad de Huelva)

Dirección: Ana Rivero García y Nicolás de Alba Fernández Secretaría de edición: Elisa Navarro Medina

\section{Consejo editorial}

José Félix Angulo Rasco. Universidad de Cádiz Rosa Ma Ávila Ruiz. Universidad de Sevilla Pilar AzcárateGoded. Universidad de Cádiz Juan Bautista Martínez Rodríguez. Universidad de Granada

Nieves Blanco García. Universidad de Málaga Fernando Barragán Medero. Universidad de La Laguna

José Carrillo Yáñez. Universidad de Huelva José Contreras Domingo. Universidad de Barcelona.

Luis C. Contreras González. Universidad de Huelva Ana $\mathbf{M}^{\mathbf{a}}$ Criado García-Legaz. Universidad de Sevilla Rosario Cubero Pérez. Universidad de Sevilla José $\mathbf{M}^{\mathbf{a}}$ Cuenca López. Universidad de Huelva Jesús Estepa Giménez. Universidad de Huelva Rafael Feito Alonso. Universidad Complutense (Madrid)

Francisco José García Gallardo. Universidad de Huelva

Soledad García Gómez. Universidad de Sevilla J. Eduardo García Díaz. Universidad de Sevilla
Fernando Hernández Hernández. Universidad de Barcelona

Salvador Llinares Ciscar. Universidad de Alicante Alfonso Luque Lozano. Universidad de Sevilla Rosa Martín del Pozo. Universidad Complutense (Madrid)

José Martín Toscano. IES Fernando Herrera (Sevilla) Jaume Martínez Bonafé. Universidad de Valencia F. Javier Merchán Iglesias. Universidad de Sevilla Emilia Moreno Sánchez. Universidad de Huelva. Rosario Ortega Ruiz. Universidad de Córdoba Antonio de Pro Bueno. Universidad de Murcia Fco. de Paula Rodríguez Miranda. Universidad de Huelva

Pedro Sáenz-López Buñuel. Universidad de Huelva Antoni Santisteban Fernández. Universidad Autónoma (Barcelona)

Emilio Solís Ramírez. Catedrático de IES. $\mathbf{M}^{\mathbf{a}}$ Victoria Sánchez García. Universidad de Sevilla. Magdalena Suárez Ortega. Universidad de Sevilla

\section{Consejo asesor}

Manuel Área Moreira. Universidad de La Laguna

Jaume Carbonell. Director Cuadernos de Pedagogía. Barcelona César Coll. Universidad de Barcelona Christopher Day. Universidad de Nothingham. U.K.

Juan Delval. Universidad Nacional de Educación a Distancia

John Elliott. Universidad de East Anglia. Norwich. U.K.

José Gimeno Sacristán. Universidad de Valencia

André Giordan. Universidad de Paris VII y Ginebra

Francisco Imbernón. Universidad de Barcelona

Ángel Pérez Gómez. Universidad de Málaga

Rafael Porlán Ariza. Universidad de Sevilla

Francesco Tonucci. Instituto de Pedagogía del C.N.R. Roma

Jurjo Torres Santomé. Universidad de A Coruña 
